\title{
Emotional Experiences of Person as a Reason of Psychosomatic Risk Origin
}

\author{
Galina Ivanovna Efremova ${ }^{1}$, Galina Valentinovna Timoshenko ${ }^{1}$, Elena Anatolievna Leonenko ${ }^{1} \&$ Irina \\ Aleksandrovna Bochkovskaya ${ }^{1}$ \\ ${ }^{1}$ The North Caucasus Federal University, Russian Federation \\ Correspondence: Galina Ivanovna Efremova, The North Caucasus Federal University, 355029, Stavropol, \\ Pushkina street, 1, Russian Federation. E-mail: efremova_ncfu@mail.ru
}

\author{
Received: September 4, 2014 Accepted: September 22, 2014 Online Published: October 30, 2014 \\ doi:10.5539/ass.v10n22p263 URL: http://dx.doi.org/10.5539/ass.v10n22p263
}

\begin{abstract}
This article is focused on issues of psychological conditions for complex research of psychosomatic disorders risk. It investigates level of a scientific development of significant categories of a longstanding uncompensated stress and emotional experiences causing it. Here is described necessary model of development of theoretical grounds of psychological research that investigates the reasons of risk of various psychosomatic disorders.
\end{abstract}

Keywords: emotional experiences, emotional stress, longstanding uncompensated stress, psychosomatic disorders risk

\section{Introduction}

Psychosomatic disorders at the present time count form $45 \%$ to $85 \%$ of all disorders amount (according to WHO's report). Such patients are unsuspicious of true nature of their disorders and continue to be treated for many years at general practitioners that in most cases doesn't lead to improvement of their state. In this situation, general practitioners also aren't always capable to recognise the psychosomatic nature of this or that disorders and to refer the patient to the corresponding specialist. Moreover, at present time in world practice effective and technological system of treatment of similar disorders doesn't exists. Such situation is directly connected with absence of the universal and complete theoretical conception of the system analysis fpr identification of the reasons of formation of psychosomatic risk which would allow to develop system of the effective psychotherapeutic help to the persons having psychosomatic disorders.

\section{Emotional Stress as a Main Psychosomatic Risk Factor}

It should be noted that, proceeding from the essence of psychosomatic defect, such conception should have to be based not only on biological and medical, but also on psychological researches. However the researches of this subject existing at the moment generally belong to representatives of natural and medical sciences (Beresin, 1988), Gindikin V. I. (2000), Isaev D. N. (2004), Karvasarskiy B. D. (1990), Oganov R. G. (2004), Olbinskay L. I. (2004), Smulichev A. B. (2004), Strukovskay M. V. (1986), Topolyanskiy V. D. (1986). In addition to the above the emotional stress is allocated by majority of researchers as an independent type of stress, genetically different of stress physiological (Kassil, 1975), Lazarus R. (1970), Rusalova M. N. (1979), Kitaev-Smik L. A. (1983), Alexandrovskiy J. L. (1997). As Kameneckiy D. A. writes, the stressful factor in general is independent risk factor for development of all disorders having the psychosomatic nature (1). From the viewpoint of Koristov J. N. the emotional stress is the main reason of cardiovascular defeats, the neuroses, some diseases of stomach and intestines, it increases probability of infectious diseases and complicates their course" (2). In her book Novikova A. I. considers psychoemotional stress as a maim risk factor of progressing arterial hypertension, coronary heart disease, stomach ulcer of a 12-perstny gut and diabetes of the II type (3). Sudakov A. V. writes, that emotional stress is marked by generalized distribution of the sympathetic and parasympathetic excitation bringing one individuals to deterioration of cardiovascular system activity, others to an ulceration of a gastrointestinal path (4). Therefore origin of psychosomatic risk is closely connected with emotional stress-i.e. the stress caused by these or those emotional experiences, instead of the fact of existence of objectively stressful situation. Furthermore, natural-science researches reports indicate the relationship of psychosomatic disorders origins and previous stay of a person in a situation of a longstanding uncompensated stress. For example, Topolyanskiy V. D. and Strukovskya M. V. note that if a process of mental adaptation is insufficiently effective, 
if process of mental adaptation is insufficiently effective, physiological components of emotions under emotional stress gain pathogenetic effect in formation of psychosomatic problems. The psychosomatic reaction of the personality on stress or frustration in a large measure is connected with chronic influence of frustration and a stress (5). From Beresin's F.B. point of view, emotional stress is indicated when an emotion gets such force or duration that an ability of a person to restoration of mental balance by means of resolving a stressful situation is insufficient (by changing the situation or changing attitude toward it). Process of resolving a stressful situation is essence of the mental adaptation that means existence of connection between probability of emotional stress origin as a longstanding and uncompensated and tendency of the person to experience of different emotions (6). This conclusion gives the grounds in the course of psychological investigation of a problem of determinacy of psychosomatic risk to place the main emphasis on studying of those personal disorders which, on the one hand, cause violation of mental adaptation, and on another-define tendency of the person to experience different potentially stressful emotions. However for implementation of system psychological research of the reasons of psychosomatic risk formation requires such description of emotional stress origin which would allow to investigate the main determining factors, and to develop complex system of prevention and psychomedical care to the persons, suffering psychosomatic disorders.

Unfortunately, in the modern psychological researches, dedicated to stress issues is not valuable for practice theoretical substantiation of the process of emotional stress formation. All psychological researches it is possible to divide into two groups. To the first group belong academic researches which don't give real opportunities for their implementation in medical and psychotherapeutic practice. For example, researches stress factors unlike with their classical behavioral explanation, which relies on external determination, consistently bring to understanding of that the stress is developed in time in the psychological space, defined by meanings and personal values (Lasarus, 1980; Vasiluk, 1984; Jaspers, 1997; Antsiferova, 1999; Janoff-Boulemane, 2001; Bek, 2002; Nurkova \& Vasilevskay, 2003; Korjova, 2004; Magomed-Aminov, 2005; Trubitsyna, 2005; Maddi, 2006; Leontiev, 2007.). Leonova A.B. defines three main approaches to the analysis of researches of a psychological stress: ecological, transactional and regulatory approaches. Ecological approach corresponds to the level of the macroanalysis of manifestations of a stress and gives the complete description of risk factors in terms of the cause-effect relationships. Transactional approach is directed on the analysis of level of an intermediate mediation of stress by individual and psychological features of the person and personal experience of overcoming of stressful situations. Regulatory approach realizes microanalysis level for accomplishment of missions and psychological mechanisms of adaptation to stressful conditions (7). Another group represents researches the researches dedicated to specific questions within a subject of emotional stress. Within neuropsychological approach (to the problem of individual distinctions) specific features of reactions to stressful situations of persons with different profiles of the lateral organization were studied $(8,9)$. Works of R. A. Karasek were devoted to creation of the general theory of a stress at workplace $(10,11)$. On the ground of a control problem researches he constructed a hypothesis that experience of a stress arises at interaction between two factors-responsibility and control. In the research of the stress caused by interaction of the individual with equipment, Bodrov V. A. claims that any professional activity can generate the psychological reasons of a stress (12). There are researches of professional stress, organizational stress, ecological stress, family stress and "stress in individual life", processes of deterioration of activity at a stress, etc. The huge number of an empirical material about various private characteristics of a stress as psychological phenomenon is saved up-however this data can't be interpreted within any universal conception because of absence that. What that the question, for example, about by means of what mechanisms the stress which is initially adaptive reaction of organism, becomes long and uncompensated, it is possible to consider open.

Therefore a large number of theoretical and empirical natural science researches were devoted to communication "emotional experience-a longstanding stress-psychosomatic risk". Moreover, the fundamental conclusion that formation of psychosomatic risk is inseparably linked with tendency of the person to these or those emotional reactions was drawn (6). However during psychological research of this relation, which could give the chance to reveal the risk of developing of these or those psychosomatic disorders at a person, there is a number of difficulties arise. These difficulties are defined by an insufficient readiness as subjects of the mechanism of a longstanding uncompensated stress origin, and a subject of substantial classification of various emotional experiences.

\section{The Existed Classification of Emotional Experiences as a Basis for Research into the Causes of Psychosomatic Risk}

Based on data of psychophysiological studies confirming the link between the tendency of the person to experience specific emotional reactions (13), it can be argued that for development of a theoretical framework 
for a fair description of the laws of a particular occurrence of psychosomatic risk is strictly necessary classification of existing emotional experiences.

Only with such a classification can be to find answers to the following questions significant for adequate description of the formation of psychosomatic risk:

- what specific emotions can be considered forming a longstanding noncompensated stress;

- how empirical study of these emotions should to be organized;

- what external and internal factors induce the person to experience such "stressful" emotions;

- how it should be organized an empirical study of the propensity of a particular person to a particular type of nosology psychosomatic reactions.

Creation of such a classification is greatly complicated by the two objective facts. Firstly, in various languages, the number of words indicating emotional experience, significantly differs. This means, that allocation of their qualitative content not depending on cultural features and caused by only objective psychophysiological components, is interfaced to very considerable difficulties. Secondly, the very lexical content of each emotional experience, determines the possibility of the use of the appropriate verbal sign, is also not well defined-that, in turn, creates serious problems for theoretical study.

As a result, the actual level of a problem of quality content of different emotional feelings at the moment cannot be considered sufficient for creation such classification. In the literature, there are a considerable number of classifications, but none of them does not solve the problem stated above, significant for the theoretical and empirical study of the formation of specific psychosomatic risk. Thus in the psychology nevertheless there is some initial theoretical groundwork for the creation of the necessary classification: K. Izard in his study formulated required properties of basic emotions:

- such emotions have distinct and specific neural substrates;

- they are indicated by means of an expressive and specific configuration of muscular movements of the person (mimicry);

- them necessarily follow a clear and specific experience, which is recognized by person;

- such emotions should be the result of evolutionary and biological processes;

- they must have an organizing and motivating effect on the person, to serve his adaptation.

However, K. Izard, naming ten basic emotions (anger, contempt, disgust, sorrow, suffering, fear, guilt, interest, joy, shame, surprise), recognizes that certain emotions, referred them to the base, do not have all of these symptoms. Thus, the emotion of guilt does not have a clear mimic and pantomimic expression (14). Close to this position stay division of emotions created by R. Plutchik, who divides emotions on primary and secondary (the latter is meant a combination of two or more primary emotions). Thus, for secondary emotions he carries the pride (anger + joy), love (joy + acceptance), curiosity (surprise + acceptance), modesty (fear + acceptance), and so on. While his classification contents (besides the emotions) personal qualities (modesty), and even acceptance (15).

Classification of Vilunas V. K. (16) divides the emotions into driving and situational. Leading emotions signalize the unmet needs and encouraged to search the target. Situational emotions arise as a result of assessments stages of behavior and encourage any act in the same direction, or change the behavior. Situational emotions are divided into three groups:

- acknowledged success -unsuccess;

- anticipating success-unsuccess;

- generalized success-unsuccess.

Easy to see that such classification does not give any reason for the systematization of emotional experiences in their substantive content, or for exploring the human propensity to experience these or other of them.

Simonov's P. V. classification is based on the nature of the action-overcoming, defense, aggression. For those emotions, that do not fit into this classification he allocates quantitative characteristics of size of requirements and an assessment of its satisfaction.

And those emotions which can't enter neither the first, nor in the second group Simonov P. V. considers as the sum of two emotions (for example, contempt $=$ disgust + anger) (17). 
Other classifications are based on allocation of any certain signs and aren't exhaustive.

For example, В. Вундт identified three characteristics of emotions: hedonic tone, or the sign of emotion (positive or negative), ready for action (relaxation-tension) and the level of activation (calm-excited) (18).

G. Shlosberg classified emotions on the basis of acceptance-repulsion (19).

In Roseman's work seventeen emotions are defined by various combinations of seven signs: expected-unexpected, consecutive-inconsistent, notwishing-wishing, high probability-low probability, high-low control, characteristic-uncharacteristic source of a problem, connection of a problem with an environment or with a person (20).

\section{The Proposed Classifications of Emotional Experiences}

In addition, all these works don't consist approaches to the strong separation of different types of emotional experiences which are presented, nevertheless, in the literature (basic emotions, emotions, feelings, attitudes, states). It can be assumed that the mere selection of similar types of emotional experiences associated with different ways of experiencing a human of the same quality specific emotional content. And as for the formation of psychosomatic risk precisely prolonged stress is significant, the creation of such a classification of emotional experiences (which would make it possible to study the process of formation of psychosomatic risk) presupposes the need to describe various types of such experiences.

Therefore, as a first necessary step towards the creation of such kind of classification, we propose the following classification of the types of emotional experiences: emotions, feelings, relationships, and states. The easiest way is to define the states: they can be defined as a scalar emotional experiences perceived by person as subjectively uncontrollable. Scalarity here (by analogy with math) is understood as a lack of focus on the experiences of anyone or anything. All other phenomena of a human emotional sphere, as we will show below, are by nature vector, it emanate from the person himself and something directed to.

It is important to note that all the words that describe the phenomena of the emotional sphere of a person (except already mentioned states), when used require some additions: admire someone despise someone to grieve for someone, etc. We call this characteristic focused on a certain subject experiences belonging to the category of feelings, victory.

In turn, the characteristics necessary to distinguish feelings and relationships, can be regarded as the degree of stability: some emotional experiences can be easily changed under the influence of the slightest change of the object to which they are directed, while others remain unchanged for a long time, regardless of any changes in this subject. For example, a certain joy at the event is able to instantaneously disappear if you change some of the characteristics of the event. At the same time, a person can be saved even if the changes respect the idea of it-until such time as such changes do not reach a certain critical level.

Based on these two characteristics, we propose to define a sense as a vector experience, changing after a single change the subject, calling it, and the emotional attitude-as a vector experience robust to single changes in object at which it is directed. It is clear that the latter definition is only suitable for those relations that can be called emotional-such as contempt or trust. At the same time, it cannot extend to social relations, that are also very important in the context of the study of personal position in a social interaction-such as, for example, impact usage, subordination, preference, etc. Such relationships may not have a component, which could be defined as an experience, and appear at the same time only in the form of some specific effects. We can say that the emotional and social relations are connected only by understanding of the relationship as a property, wich arises and exists only in the interaction of two or more fragments of reality.

However, from our point of view, any classification of this kind can be regarded as a strict only if this separation will be allocated an objective criterion. Therefore, in our study for the classification of the various phenomena of the emotional life, we propose to use the linguistic characteristics of words designating the Russian language in the manifestation of the emotional sphere of person.

We estimate several dictionaries of the Russian language for the purpose of identify the nouns that describe the phenomena of human emotional sphere. In total, it has been found about eighty of these nouns (except obsolete and rarely used). They were divided from a linguistic point of view, in three different groups.

The first group of nouns is characterized by the inability to form from them a verb in the active voice (inspiration, guilt, curiosity, etc.). This means that the person in this case does not take such their emotional experience as a result of their own actions. 
Nouns belonging to this group have and other expressed characteristics of usage: about them, you can either say "stay in inspiring", "be confused", etc., or to form a passive participle of them-"encouraged", "confused". Accordingly, from a linguistic point of view, these words referred to the phenomena of the emotional sphere just act as scalar experiences that have a grammatical passive voice.

Strictly speaking, this scalarity is subjectively perceived-in the sense that a person does not consider himself the source of this condition. The second group includes nouns, which can be formed to the verb in the active voice, characterized by the recurrence category (anger-to be angry, joy-to be enjoy, shame-ashamed, etc.). Category of recurrence in Russian language reflects some specific contents, one of which-the focus some action on the subject of this action.

This characteristic, in our opinion, gives to us the reason to include nouns of the second group to category of feelings as emotional actions which on the one hand, are initiated and authorized by the person, and the other-are directed to him by himself.

The third group selected by us are nouns, from which it is possible to form an irreflexive verb in the active voice (disgust-I disgust, malevolence-I gloat, regret-I regret, etc.).

Proceeding from grammatical characteristics, it is possible to tell that similar nouns describe such phenomena of the emotional sphere of person which are fully initiated and authorized by him, but are directed-unlike feelings-outside.

From this point of view, we propose to consider the nouns belonging to the third group, the relationships. Separate consideration should be paid to the division of the terms which often are used as synonyms,-emotions and feelings. In addition, this issue is closely adjacent to the theme selection of basic emotions-as well as the issue of whether it is worth to divide emotions and basic emotions.

In the proposed classification to separate emotions and feelings we use their specificity for human. The experience of some concrete emotions is similar-to external displays and the context of their occurrence-for humans and higher mammals. To these emotions we may include indifference, curiosity, joy, anger, fear and sadness. All these emotions precisely correspond to the vital needs of both animals and humans:

- indifference is typical for situations where none of the perceived stimulus is not perceived neither as positively significant nor as negatively significant, and therefore energy costs represented to an individual unreasonable;

- curiosity arises in situations where there is some unclear stimulus that triggers an orienting reflex;

- joy is experienced in situations where there is an opportunity to fill some urgent need;

- anger is experienced in situations where filling the urgent needs something or someone interferes;

- fear is experienced in situations in which there is a threat to life or physical integrity;

- sadness arises in situations in which there are no any opportunity to fill some urgent needs.

This description, from our point of view, provides enough opportunities to explore the functions of the emotional sphere in the formation of various psychosomatic risks (21).

\section{Conclusion}

Conclusion. All of the aforesaid leads us to draw some conclusions about the state of the problem of psychosomatic risk determination at the present time.

Firstly, we should note the absence in the psychological literature of such kind of definition of stress, which could identify the psychological content of this term-in contrast to the comprehensive studies of its physiological content. It was shown above that the existing division of stress on physiological and emotional does not contain a concrete phenomenological and psychological differentiation and justification for such allocation. In the modern psychology there is no a model of origin and continued existence of emotional stress, which would be consistent with the requirements to model as a tool for scientific knowledge.

Such requirements, which formulated the epistemology suggest that the model should on the one hand reflect all the most significant characteristics of the object being modeled, and on the other-to give additional possibilities to study it.

This means that the required model should allow to describe those mechanisms of origin and continuation of emotional stress, which cannot be deduced from the external manifestations of this stress.

Further, the development of ideas about the emotional stress suggests the classification of human emotional experience to their substantive content and the method of their experiences. 
In addition, it should be noted that the mere principle of systemacity as one of the basic methodological principles of psychology assumes the integrity of a way of explaining the mental phenomenon.

Consequently, a further description of the language of psychology requires a mechanism for the formation and psychosomatic illness as a result of prolonged emotional stress.

Therefore, the mechanism of formation of a psychosomatic disorder as a result of longstanding emotional stress requires additional development in psychology.

And finally, the issue what makes a stress longstanding and emotional uncompensated still stays unclear. Impossible to confirm without further justification, that emotional stress (as opposed to physiological stress) certainly is not constructive, and the more maladaptive. As a preliminary hypothesis can be assumed that a person's ability to find a constructive way out of stressful situations affect some features of his personality. That is, for a comprehensive study of topics of interest to us is necessary to identify the personality features that determine the possibility of uncompensated stress origin-and, consequently, the formation of psychosomatic risk.

Thus, we can conclude that for the psychological study of problem individual person's predisposition to the origin of a psychosomatic risk previously required to solve several theoretical problems.

The first of these problems-the need for a rigorous and sophisticated definition of emotional stress, which would provide the essential criterion for distinguishing between adaptive and non-adaptive stress.

The second task-to create a classification of human emotional experiences that addresses the following reasons:

- the qualitative content of experience;

- the way of his experience (or quantification of its contents);

- the reason for its origin.

The third task-to create a model of origin and existence of emotional stress, which would give the opportunity to:

- to propose a criterion for distinguishing between constructive and unconstructive emotional stress;

- to describe the differences in the causes and mechanisms responsible for the adaptive emotional stress and emotional stress, with the characteristics of duration and uncompensated;

- to identify the emotional and personal characteristics of a person that influence the characteristics of a stress typical for him.

Only after the solution of these three tasks the main task can be solved-the task of creating a psychological model of the formation of psychosomatic risk that meets the following requirements:

- would allow to explain the mechanism of formation of such risk;

- would give the chance to describe the reasons of formation of various psychosomatic risks;

- would create a basis for the development of appropriate diagnostic techniques.

Thus, a significant empirical study on the psychological reasons for the formation of psychosomatic risk cannot be carried out before the necessary theoretical base will be provided.

In our opinion, the absence of such theoretical conception explains the lack of elaboration of this issue, the practical value of which is difficult to overestimated.

In our opinion, absence of such theoretical concept explains insufficiency of a readiness today this subject which practical value it to overestimate.

This article is prepared under the Task No. 25.1679.2014/K of 11.07 .2014 on performance of research work within the framework of the project of the state assignment in the field of scientific activity.

\section{References}

Berezin, F. B. (1998). Psychological mechanisms of psychosomatic diseases. Russian Medical Journal, 2, 4-49.

Bodrov, V. A. (2000). Information Stress: Textbook for universities. M.: PER SE.

Ilyin, E. P. (2001). Emotions and feelings. St. Petersburg.: "Piter".

Izard, K. E. (1980a). Human Emotions, 39-40.

Izard, K. E. (1980b). Human Emotions, 40-41.

Kamenetsky, D. A. (2001). Neurosology and psychotherapy. M.: Helios ARV. 
Karasek, R. A. (1979). Job demands, job decision latitude, and mental train: Implications for job redesign. Administrative Science Quarterly, 24, 285-308. http://dx.doi.org/10.2307/2392498

Karasek, R. A., \& Theorell, T. (1990). Healthy Work. Basic Books. New York.

Khomskaya, E. D. (1997). In E. D. Khomskaya, I. V. Efimova, E. V. Budyka, \& E. V. Enikolopova (Eds.), Neuropsychology of individual differences. M.: Publishing house of the RPA.

Leonova, A. B. (2003). Department of Psychology of Moscow State University named after M. V. Lomonosov, Moscow, Russia. "The main theoretical approaches and research methodology of psychological stress". VII Interdisciplinary Conference on Biological Psychiatry "Stress and Behavior".

Moskvin, V. A. (1988). Hemispheric asymmetry and individual styles of emotional response. Questions of psychology, 6, 116-120.

Novikova, A. I., Sidorov, P. I., \& Solovyev, A. G. (2006). Psychosomatic Medicine. M.: MEDpress-inform.

Roseman, I. J. (1996). Appraisal Determinants of Emotions: Constructing a More Accurate and Comprehensive Theory. Cognition \& Emotion, 10(3). http://dx.doi.org/10.1080/026999396380240

Shcherbatyh, Y. V. (2008). The psychology of stress and methods of correction (p. 89). St. Petersburg: "Piter“.

Simonov, P. V. (1981). Emotional brain. M.: Science.

Sudakov, K. V. (1976). Emotional stress and hypertension. New York: Mc-Graw-Hill.

Timoshenko, G. V., \& Leonenko, E. A. (2011). Metaphorical psychotherapy. M.: Psychotherapy.

Topolyansky, V. D., \& Strukovskaya, M. V. (1986). Psychosomatic disorders. M., Medicine.

Vilyunas, V. K. (1990). Psychological mechanisms of human motivation. M.: Publishing House of Moscow State University.

Wundt, W. (1880). Fundamentals of physiological psychology. Feelings and affects. St. Petersburg.

\section{Copyrights}

Copyright for this article is retained by the author(s), with first publication rights granted to the journal.

This is an open-access article distributed under the terms and conditions of the Creative Commons Attribution license (http://creativecommons.org/licenses/by/3.0/). 